\title{
Improvement of the ephemerides of Phoebe, 9th satellite of Saturn, from new observations made from 1995 to 2000
}

\author{
J.-E. Arlot, A. Bec-Borsenberger, A. Fienga, and N. Baron \\ Institut de Mécanique Céleste et de Calcul des Éphémérides, observatoire de Paris, UMR 8028 du CNRS, \\ 77 avenue Denfert-Rochereau, 75014 Paris, France \\ e-mail: arlot@imcce.fr
}

Received 11 July 2002 / Accepted 27 February 2003

\begin{abstract}
In order to improve the model used for the ephemerides of Phoebe, the 9th satellite of Saturn, we started observations in 1998. We made 135 observations in 1998 and 39 observations in 1999 using the $120 \mathrm{~cm}$-telescope of Observatoire de HauteProvence, France. We used a numerical integration in order to calculate new initial conditions and to be able to build new ephemerides. We also used some precise observations made from 1995 to 2000 together with old observations for that purpose. The result is a decrease in the uncertainties on Phoebe's orbit.
\end{abstract}

Key words. planets and satellites: individual: Saturn, Phoebe - Astrometry

\section{Introduction}

The theoretical model and the ephemerides of the motion of the 9th satellite of Saturn, Phoebe, used at IMCCE (Institut de Mécanique Céleste et de Calcul des Éphémérides) and distributed through the Web, needs improvement because of the few astrometric observations made since its discovery in 1904 and of the decrease of the accuracy of the ephemerides. We started a campaign of observation and used the data obtained for the determination of a new ephemeris. This paper provides the results. The set of data, together with some explanations of the problems encountered during the reduction of the data, are provided by Fienga et al. (2002).

\section{The theoretical model for the Phoebe's orbit}

The model used to determine the Phoebe's orbit is the same as that determined in 1982 (Bec-Borsenberger and Rocher) i.e. a numerical integration of the motion of Phoebe around Saturn mainly taking into account the perturbations by the Sun, Jupiter, Uranus, Neptune and Titan that are sufficient for the fit to the observations; perturbations by the other major planets, the Earth, Mars, Uranus are also taken into account, but as the perturbations due to the other satellites and the oblateness of Saturn, these perturbations are negligible since they are two orders smaller (Jacobson 1998).

Send offprint requests to: J.-E. Arlot, e-mail: Jean-Eudes. Arlot@imcce.fr

* Based in part on observations made at observatoire de Haute Provence (CNRS), France.
The calculated ephemeris of Phoebe is obtained by the predictor-corrector numerical method of Bulirsh \& Stoer (1966), using the code written by Balmino in 1969.

For the computation of the perturbations by major planets and of the geocentric position of Phoebe, the SLP96 ephemeris of the IMCCE is used. This ephemeris is extracted from VSOP87 (Bretagnon \& Francou 1988) and from ELP2000/82 (Chapront-Touzé \& Chapront 1988). Since the SLP96 position error is about $0.2^{\prime \prime}$ for Saturn, this will limit the precision of the geocentric calculated positions of Phoebe. The perturbations by Titan are computed using its positions derived from Dourneau's theory (1987).

The equations of motion are written in the reference system ICRS with rectangular planetocentric coordinates.

The initial conditions used are derived from the rectangular equatorial saturnicentric coordinates in the reference system 1950.0, published by Bec-Borsenberger \& Rocher (1982), for the epoch JD 2440600.5 TT (14 January 1970, 0h TT) in astronomical units (au) and astronomical units per day (au/d):

$$
\begin{aligned}
& x=-0.08170190500 \mathrm{au} \\
& y=+0.03581353800 \mathrm{au} \\
& z=+0.02645037400 \mathrm{au} \\
& \dot{x}=+0.00029562070 \mathrm{au} / \mathrm{d} \\
& \dot{y}=+0.00079581937 \mathrm{au} / \mathrm{d} \\
& \dot{z}=+0.00034197713 \mathrm{au} / \mathrm{d} .
\end{aligned}
$$

These values have been obtained by fitting the computed orbit using the DE102 ephemeris (Newhall et al. 1983) to 182 observed positions of Phoebe, from 1904 to 1982.

From these values and for the initial epoch JD 2440600.5 , new sets of initial conditions were determined that included 
Table 1. Results (in arcseconds) after fitting the observations.

\begin{tabular}{lllllll}
\hline \hline & Set (1) & \multicolumn{3}{c}{ Set (2) } & \multicolumn{3}{c}{ Set (3) } \\
& $\alpha$ & $\delta$ & $\alpha$ & $\delta$ & $\alpha$ & $\delta$ \\
\hline$\epsilon$ & 0.67 & 0.82 & 0.09 & 0.07 & 0.02 & 0.04 \\
$\sigma$ & 1.16 & 1.16 & 0.51 & 0.45 & 0.27 & 0.27 \\
\hline
\end{tabular}

the 1904-1982 observations of Phoebe and five recent observations taken in 1989 with the CERGA Schmidt telescope and referred to ICRS. The DE403 ephemeris of the Jet Propulsion Laboratory was used instead of its older version, DE102, in this analysis:

$$
\begin{aligned}
& x=-0.082224770685306921 \mathrm{au} \\
& y=+0.03492568817671920 \mathrm{au} \\
& z=+0.02603360967357215 \mathrm{au} \\
& \dot{x}=+0.0002852481501216026 \mathrm{au} / \mathrm{d} \\
& \dot{y}=+0.0007992545736380831 \mathrm{au} / \mathrm{d} \\
& \dot{z}=+0.0003429959321994923 \mathrm{au} / \mathrm{d} .
\end{aligned}
$$

For this determination, denoted "set (1)" in the following, the mean value and the root-mean-square of residuals in right ascension and declination are given in Table 1.

\section{The fit to the observations}

Our goal is to determine new initial conditions using old observations and the new ones made in 1998 and 1999 at the HauteProvence observatory together with other new precise data.

\subsection{Old observations}

For our purpose, we selected old observations to have observations spread over a long interval of time in order to model the long period terms in the motion of Phoebe. We used 167 photographic observations made from 1904 to 1989, corresponding to 118 nights of observations and to 20 oppositions of Saturn, denoted $\mathrm{P}$ in the following.

\subsection{New observations}

We used also more recent CCD observations: 7 observations made at La Palma observatory in 1995 and 1997 corresponding to 7 nights (each observation is a normal point for one night corresponding to 40 exposures) by Harper \& Beurle (Jones 1999) denoted $\mathrm{H}$ in the following and 62 observations made with the Flagstaff Astrometric Automatic Transit Telescope (FASTT) denoted S in the following (Stone 2000; Stone \& Harris 2000).

\subsection{Our observations}

To increase the number of data available, we made ourself new observations. The observations were made at Observatoire de Haute-Provence, France, using the $120 \mathrm{~cm}$-telescope. The focal length is about 7 meters. The CCD target is $1024 \times 1024$ with a scale of 0.68 arsec per pixel. The field of the target is about 12 min of a degree. The observations were reduced using

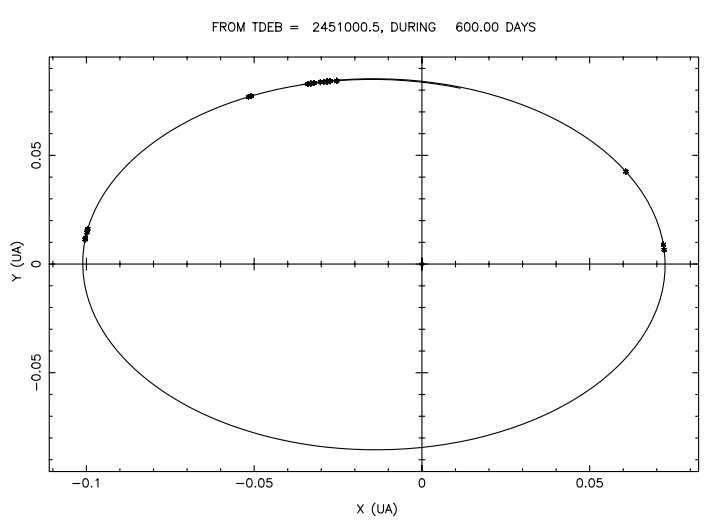

Fig. 1. OHP observations along Phoebe's orbit.

the USNO A2 catalogue with the PRIAM software (Fienga \& Berthier 2001). Zonal corrections were made with the observations of ICRF references in the fields. More details are provided in Fienga et al. (2002) together with the data themselves.

The observations were made in 1998 and 1999. 84 images were made in August 1998, 10 images were made in September 1998, 35 images were made in December 1998, 16 images were made in October 1999 and 7 images in November 1999. We obtained 129 images in 1998 and 23 in 1999, i.e. a total of 152 images corresponding to 18 nights and 2 oppositions of Saturn. The coverage of the orbit of Phoebe is presented by Fig. 1 plotting the observed right ascensions and declinations on Phoebe's orbit projected on the plane of Saturn's orbit.

\subsection{Determination of initial conditions}

In the following, the different sets of observations will be designated as indicated below by:

- P: 167 photographic observations made from 1904 to 1989;

- H: 7 CCD observations (normal points from 40 individual images) made at La Palma observatory in 1995 and 1997;

- S: 62 CCD observations made at Flagstaff observatory from 1995 to 2000 ;

- OHP: 152 CCD observations made at Haute Provence observatory in 1998 and 1999.

Starting from the set (1), a new set of initial conditions was calculated at the epoch JD 2440600.5 by fitting the orbit to the observations (1904-2000): $\mathrm{P}+\mathrm{H}+\mathrm{S}$. For this determination, the fact that the $\mathrm{H}$ observations are combined into one normal point for each night is taken into account for the weighting of the equations; furthermore, the CCD observations are considered four orders more accurate than the photographic observations. The set (2) obtained in this way is:

$$
\begin{aligned}
& x=-0.08222700864052486 \mathrm{au} \\
& y=+0.03486157617620074 \mathrm{au} \\
& z=+0.02605312908514955 \mathrm{au} \\
& \dot{x}=+0.0002849371122126130 \mathrm{au} / \mathrm{d} \\
& \dot{y}=+0.0007993528677276216 \mathrm{au} / \mathrm{d} \\
& \dot{z}=+0.0003432878745894294 \mathrm{au} / \mathrm{d} .
\end{aligned}
$$

From this second set, another set of initial conditions was calculated at the same epoch by adding the OHP observations to 
Table 2. Statistics for different fits.

\begin{tabular}{llllll}
\hline \hline & & \multicolumn{2}{c}{$\mathrm{P}$} & \multicolumn{2}{c}{ OHP } \\
& & $\alpha$ & $\delta$ & $\alpha$ & $\delta$ \\
\hline \multirow{3}{*}{ Set (1) } & $\epsilon$ & 0.67 & 0.82 & -1.07 & 0.42 \\
& $\sigma$ & 1.16 & 1.16 & 1.23 & 0.66 \\
& $\sigma_{\mathrm{c}}$ & 0.95 & 0.82 & 0.63 & 0.51 \\
\hline \multirow{3}{*}{ Set (2) } & $\epsilon$ & 0.61 & 0.62 & 0.14 & 0.13 \\
& $\sigma$ & 1.30 & 1.07 & 0.22 & 0.21 \\
& $\sigma_{\mathrm{c}}$ & 1.15 & 0.87 & 0.17 & 0.19 \\
\hline \multirow{3}{*}{$\operatorname{Set}(3)$} & $\epsilon$ & 0.65 & 0.60 & 0.05 & 0.08 \\
& $\sigma$ & 1.27 & 1.05 & 0.18 & 0.18 \\
& $\sigma_{\mathrm{c}}$ & 1.09 & 0.86 & 0.18 & 0.16 \\
\hline
\end{tabular}

the previous sets of observations. The resulting initial conditions are the following:

$$
\begin{aligned}
& x=-0.08223147387792590 \mathrm{au} \\
& y=+0.03485873123707742 \mathrm{au} \\
& z=+0.02605313481431400 \mathrm{au} \\
& \dot{x}=+0.0002848746714494925 \mathrm{au} / \mathrm{d} \\
& \dot{y}=+0.0007993482102968699 \mathrm{au} / \mathrm{d} \\
& \dot{z}=+0.0003432686704878827 \mathrm{au} / \mathrm{d} .
\end{aligned}
$$

The statistics (mean values and root-mean-squares of the residuals) resulting from these determinations are given in Table 1.

\subsection{Residuals, mean errors and root-mean-square of the residuals}

Table 2 provides for each set of initial conditions (1), (2), (3) the mean error $\epsilon$, the root-mean-square of the residuals $\sigma$, and the standard deviation from the mean $\sigma_{\mathrm{c}}$, for the photographic observations $\mathrm{P}$ used for the present ephemeris published by IMCCE, and for the OHP observations used for the improvement of the ephemeris.

One will find in Fienga et al. (2002) the analysis of the observations. The plot of $(\mathrm{O}-\mathrm{C}) \mathrm{s}$ for ephemerides built from set (2) are shown in Fig. 2 to display the precision of the observations. Table 2 allows us to know the precision of the different ephemerides.

\section{Results and discussion}

The new ephemerides that we built using a numerical integration with initial conditions fitted on the sets of observations that we presented here represent the motion of Phoebe with better accuracy than previous ones. We see that: - the old observations present the same $\epsilon, \sigma$ and $\sigma_{\mathrm{c}}$ for the 3 fits; - the new OHP observations have large $\epsilon, \sigma$ and $\sigma_{\mathrm{c}}$ for the fit corresponding to set (1); they have their $\epsilon, \sigma$ and $\sigma_{\mathrm{c}}$ improved with the fit corresponding to set (2) including recent observations and have small $\epsilon, \sigma$ and $\sigma_{\mathrm{c}}$ when they are itself used in the fit (set (3)). In the last case, the $\epsilon, \sigma$ and $\sigma_{\mathrm{c}}$ are not degraded for old observations, which means that old observations, even though not precise enough, help improve the accuracy of the ephemerides.
(O-C) in right ascension

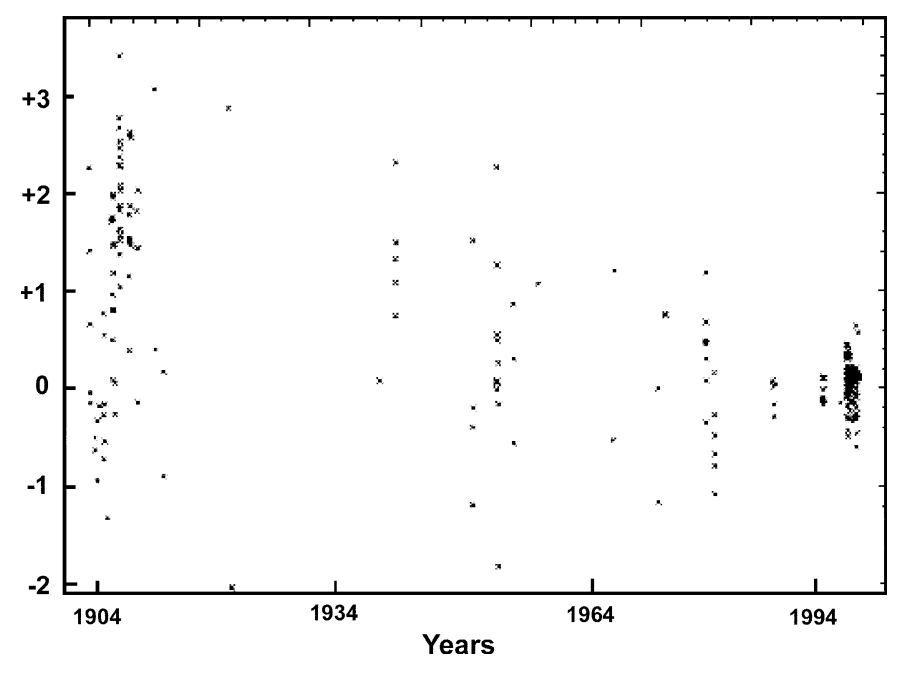

(O-C) in declination

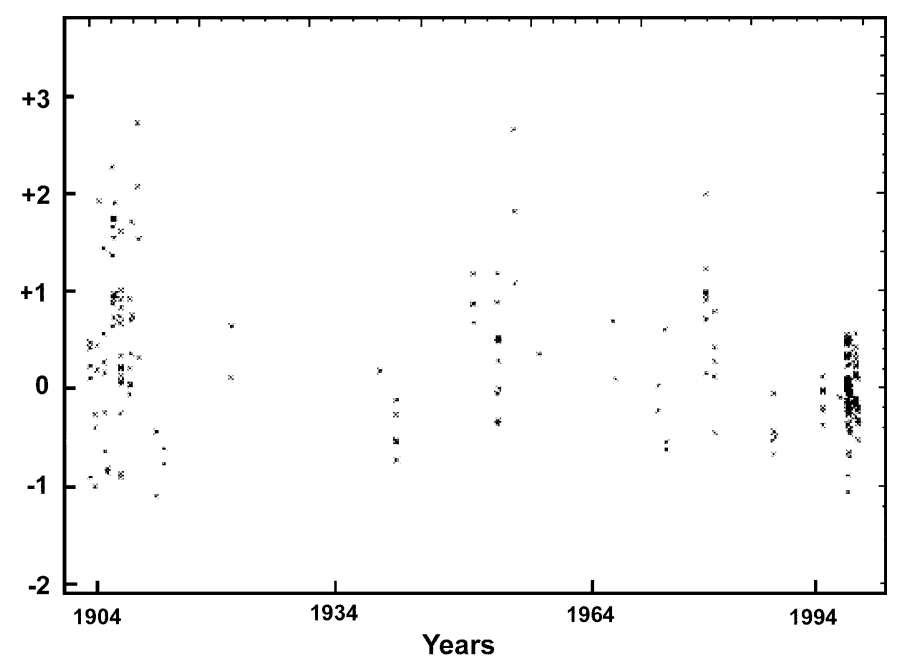

Fig. 2. O-C's in right ascension and declination for the whole set of observations from 1904 to 2000.

Our goal is to be able to extrapolate the ephemerides with sufficient accuracy that will be measured in future observations.

In the paper by Fienga et al. (2002), it is shown that any improvement of the star catalogue used for the reduction of the observations help greatly to increase the level of accuracy of the observations and then the ephemerides built from them. Future observations should be reduced using the UCAC catalog for this purpose.

\section{Conclusion}

Our work shows that we need to gather more observations and to make new ones in order to be able to get ephemerides that we can extrapolate to have positions of Phoebe in the next years with sufficient accuaracy. Accurate observations linked to ICRF sources will permit one to improve the dynamical model of the motion of Phoebe by analyzing the $(\mathrm{O}-\mathrm{C})$ s and consequently the motion of Saturn itself, the astrometric observations of which are very difficult to make. 
Acknowledgements. This work has been made possible thanks to the CNRS (Centre National de la Recherche Scientifique) and to the Institut de Mécanique Céleste et de Calcul des Éphémérides, UMR 8028 du CNRS.

We also wish to thank the staff of the Observatoire de HauteProvence (OHP) for their efforts and their help during the observational campaign.

\section{References}

Bec-Borsenberger, A., \& Rocher, P. 1982, A\&AS, 50, 423 Bretagnon, P., \& Francou, G. 1988, A\&A, 202, 30

Bulirsh, R. \& Stoer, J. 1966, Num. Math., 8, 1

Chapront-Touzé, M., \& Chapront, J. 1988, A\&A, 190, 342
Dourneau, G. 1987, Thesis, Bordeaux university

Dourneau, G. 1993, A\&A, 267, 292

Fienga, A., Arlot, J.-E., Baron, N., et al. 2002, CCD observations of Phoebe made in 1998, 1999 at Observatoire de Haute-Provence, A\&A, 391, 767

Fienga, A., \& Berthier, J. 2001, PRIAM: A new efficient astrometric software, Ceres 2001 Workshop on Astrometry and Physics of Minor Planets from Observational Networks (Paris)

Jacobson, R. A. 1998, A\&AS, 128, 7

Jones, D. 1999, private communication

Newhall, X. X., Standish, E. M., \& Williams, J. G. 1983, A\&A, 125, 150

Stone, R. C. 2000, AJ, 120, 2124

Stone, R. C., \& Harris, F. H. 2000, AJ, 119, 1985 